ANNALES

POLONICI MATHEMATICI

$87(2005)$

\title{
The Łojasiewicz exponent of subanalytic sets
}

\author{
by StanisŁaw Spodzieja (Eódź)
}

\begin{abstract}
We prove that the infimum of the regular separation exponents of two subanalytic sets at a point is a rational number, and it is also a regular separation exponent of these sets. Moreover, we consider the problem of attainment of this exponent on analytic curves.
\end{abstract}

1. Introduction. Let $\mathcal{M}$ be a finite-dimensional, real analytic manifold countable at infinity, $\varrho$ be a distance function on $\mathcal{M}$ induced by a Riemannian metric on $\mathcal{M}$, and let $X, Y \subset \mathcal{M}$ be closed subanalytic sets. In the theory of semi-analytic and subanalytic sets ([2], [15], [22], [24], [25]), an important role is played by the fact (proved by Łojasiewicz in [22] and [25]) that $X$ and $Y$ are regularly separated at any $x_{0}$. Namely:

TheOrem 1.1. For any $x_{0} \in X \cap Y$ there exist $\nu>0$ and $C>0$ such that for some neighbourhood $\Omega \subset \mathcal{M}$ of $x_{0}$,

$$
\varrho(x, X)+\varrho(x, Y) \geq C \varrho(x, X \cap Y)^{\nu} \quad \text { for } x \in \Omega .
$$

If additionally $x_{0} \in \overline{X \backslash Y}$, then $\nu \geq 1$ and $(\mathrm{S})$ is equivalent to

$$
\varrho(x, Y) \geq C^{\prime} \varrho(x, X \cap Y)^{\nu} \quad \text { for } x \in \Omega^{\prime} \cap X,
$$

where $C^{\prime}>0$ and $\Omega^{\prime}$ is a neighbourhood of $x_{0}$.

Note that the condition $x_{0} \notin \overline{X \backslash Y}$ leads to the trivial cases $\nu=0$ in $\left(\mathrm{S}^{\prime}\right)$ and $\nu=0$ or $\nu=1$ in (S), provided we put $0^{0}=0$.

In this paper we investigate the smallest exponent $\nu$ satisfying (S). Bochnak and Risler ([3, Corollary 2]) proved:

THEOREM 1.2. For a fixed relatively compact neighbourhood $\Omega$ of $x_{0} \in$ $X \cap Y$,

$$
\mathcal{L}_{\Omega}(X, Y):=\inf \left\{\nu \in \mathbb{R}: \exists_{C>0} \forall_{x \in \Omega} \varrho(x, X)+\varrho(x, Y) \geq C \varrho(x, X \cap Y)^{\nu}\right\}
$$

is a rational number.

2000 Mathematics Subject Classification: Primary 32B20.

Key words and phrases: subanalytic set, Łojasiewicz inequality, Łojasiewicz exponent. 
The exponent $\nu$ satisfying (S) for some $\Omega$ and $C>0$ is called a regular separation exponent of $X$ and $Y$ at $x_{0}$. The infimum of all regular separation exponents of $X$ and $Y$ at $x_{0}$ is called the Eojasiewicz exponent of $X, Y$ at $x_{0}$ and denoted by $\mathcal{L}_{x_{0}}(X, Y)$. It is easy to see that $\mathcal{L}_{x_{0}}(X, Y)=\inf \left\{\mathcal{L}_{\Omega}(X, Y): \Omega\right.$ a relatively compact neighbourhood of $\left.x_{0}\right\}$.

We shall prove the following generalisation of Theorem 1.2.

Theorem 1.3. Let $x_{0} \in X \cap Y$. Then $\mathcal{L}_{x_{0}}(X, Y) \in \mathbb{Q}$, and $(\mathrm{S})$ holds for $\nu=\mathcal{L}_{x_{0}}(X, Y)$, some $C>0$ and a neighbourhood $\Omega$ of $x_{0}$.

The exponent $\mathcal{L}_{x_{0}}(X, Y)$ is attained on an analytic curve, namely, we have

Theorem 1.4. Let $x_{0} \in X \cap Y$ and $x_{0} \in \overline{\mathcal{M} \backslash(X \cap Y)}$. Then for any neighbourhood $\Omega$ of $x_{0}$ there exists an analytic curve $\varphi:[0, r) \rightarrow \Omega$ such that $\varphi(0) \in X \cap Y, \varphi((0, r)) \subset \Omega \backslash(X \cap Y)$ and for some constant $C^{\prime}>0$,

$$
\varrho(\varphi(t), X)+\varrho(\varphi(t), Y) \leq C^{\prime} \varrho(\varphi(t), X \cap Y)^{\mathcal{L}_{x_{0}}(X, Y)}, \quad t \in[0, r) .
$$

The above two theorems will follow from analogous results in a slightly more general situation. Namely, for three subanalytic sets $X, Y, Z$ $\subset \mathcal{M}$ such that $X \cap Y \subset Z$, we define a regular separation exponent of $Y$ and $Z$ on $X$ at a point $x_{0} \in X \cap Y$ to be any real positive $\nu$ such that

$$
\varrho(x, Y) \geq C \varrho(x, Z)^{\nu} \quad \text { for } x \in X \cap \Omega,
$$

where $C>0$ and $\Omega$ is a neighbourhood of $x_{0}$. The infimum of all such exponents $\nu$ will be denoted by $\mathcal{L}_{x_{0}}(X ; Y, Z)$. If $Z=X \cap Y$ then obviously $\mathcal{L}_{x_{0}}(X ; Y, Z)=\mathcal{L}_{x_{0}}(X, Y)$, provided $x_{0} \in \overline{X \backslash Y}$. The main result of this paper is the following

Theorem 1.5. Let $X, Y, Z$ be closed subanalytic subsets of $\mathcal{M}$ with $X \cap Y \subset Z$, and let $x_{0} \in X \cap Y$.

(i) We have $\mathcal{L}_{x_{0}}(X ; Y, Z) \in \mathbb{Q}$, and (\#) holds for $\nu=\mathcal{L}_{x_{0}}(X ; Y, Z)$, some $C>0$ and a neighbourhood $\Omega$ of $x_{0}$.

(ii) If $x_{0} \in \overline{X \backslash Z}$, then $\mathcal{L}_{x_{0}}(X ; Y, Z)$ is attained on an analytic curve, i.e. for any neighbourhood $\widetilde{\Omega}$ of $x_{0}$ there exists an analytic curve $\varphi$ : $[0, r) \rightarrow X \cap \widetilde{\Omega}$ such that $\varphi((0, r)) \subset X \backslash Z$ and $\varphi(0) \in X \cap Y$, and for some constant $C_{1}>0$,

$$
C_{1} \varrho(\varphi(t), Y) \leq \varrho(\varphi(t), Z)^{\mathcal{L}_{x_{0}}(X ; Y, Z)} \quad \text { for } t \in[0, r) .
$$

The proof of the above theorem will be given in Section 2. Unfortunately, in Theorems 1.4 and 1.5, we cannot require that $\varphi(0)=x_{0}$ (see Example 2.5). This observation shows that, in the proof of Theorem 1.5, it does not suffice to apply the Curve Selection Lemma. We have to use another tool, the 
notion of Lipschitz stratification introduced by T. Mostowski [26] (see also $[27]-[29])$.

Sections 3 and 4 are devoted to applications of Theorem 1.5.

Let $X, Y$ be subanalytic sets, $F: X \rightarrow Y$ be a subanalytic mapping and $\Gamma(F)$ be the graph of $F$. Let $\mathcal{L}_{x_{0}}(F):=\mathcal{L}_{\left(x_{0}, y_{0}\right)}\left(\Gamma(F) ; X \times\left\{y_{0}\right\}, V \times Y\right)$, where $x_{0} \in X, y_{0}=F\left(x_{0}\right)$, and $V=F^{-1}\left(y_{0}\right)$. Theorem 1.5 implies that $\mathcal{L}_{x_{0}}(F)$ is the smallest exponent $\nu$ satisfying the following fundamental $E_{o-}$ jasiewicz inequality:

$$
\varrho\left(F(x), y_{0}\right) \geq C \varrho(x, V)^{\nu}, \quad x \in X \cap \Omega,
$$

for some neighbourhood $\Omega$ of $x_{0}$, and $C>0$ (Corollary 3.1). The inequality (Ł) plays an important role in singularity theory ([2], [19], [22], [23], [32]), and in the solution of the division problem in distribution theory ([16], [21]).

For two subanalytic mappings $F: X \rightarrow Y, g: X \rightarrow Z$, where $X, Y, Z$ are subanalytic sets, and $x_{0} \in X, y_{0}=F\left(x_{0}\right), z_{0}=g\left(x_{0}\right)$, we show that the number $\mathcal{L}_{x_{0}}(F / g):=\mathcal{L}_{\left(x_{0}, y_{0}, z_{0}\right)}\left(\Gamma(F, g) ; X \times\left\{y_{0}\right\} \times Z, X \times Y \times\left\{z_{0}\right\}\right)$ is the smallest exponent $\nu$ satisfying

$$
\varrho\left(F(x), y_{0}\right) \geq C \varrho\left(g(x), z_{0}\right)^{\nu}, \quad x \in X \cap \Omega,
$$

in a neighbourhood $\Omega$ of $x_{0}$ for some $C>0$, provided $F^{-1}\left(y_{0}\right) \subset g^{-1}\left(z_{0}\right)$ (Corollary 4.1). In particular, we obtain the Lejeune-Jalabert and Teissier result stating that in the complex analytic case $\mathcal{L}_{x_{0}}(F / g) \in \mathbb{Q}([20$, Corollary 6.4], cf. [3]). We collect some relations between $\mathcal{L}_{x_{0}}(F)$ and $\mathcal{L}_{x_{0}}(F / g)$ in Remark 4.3.

If $X$ is a semi-algebraic set and $F: X \rightarrow \mathbb{R}^{m}$ is a semi-algebraic mapping, then the set $\left\{\mathcal{L}_{x}(F): x \in V\right\}$ is finite, where $V=F^{-1}(0)$ (Corollary 2.7). So, the number $\mathcal{L}(F)=\max _{x \in V} \mathcal{L}_{x}(F)$ is the smallest exponent $\nu$ for which (£) holds at each $x_{0} \in V$. In Theorem 3.5 we prove that there exists a rational number $l$ such that

$$
|F(x)|(1+|x|)^{l} \geq C \varrho(x, V)^{\mathcal{L}(F)} \quad \text { in } X
$$

for some constant $C>0$. Moreover, the infimum $l_{\infty}(F)$ of all such exponents $l$ is also a rational number and satisfies (JKS), provided $\mathcal{L}(F)>0$ and $X \backslash V$ is unbounded (if $X$ is compact, then (JKS) holds for $l=0$ ). Theorem 3.5 is a generalisation of the Ji, Kollár and Shiffman result to the semi-algebraic case ([17, Theorem 5 and Corollary 6], see also [4], [9], [10], [18]). In the case when $V$ is finite, (JKS) is also important in the polynomial mappings theory (Remark 3.6).

In Section 4 we consider the notion of separation of two mappings. In particular we give a version of (JKS) for two mappings (Theorem 4.5, cf. [12]).

2. Separation of subanalytic sets. We recall some notions. A subset of a linear space $M$ is called semi-algebraic when it is defined by a finite 
alternative of finite systems of inequalities $P>0$ or $P \geq 0$, where $P$ are polynomials on $M$. A set $E \subset \mathcal{M}$ is called semi-analytic if every point of $\mathcal{M}$ has a neighbourhood $\Omega$ such that $E \cap \Omega$ is defined by a finite alternative of finite systems of inequalities $f>0$ or $f \geq 0$, where $f: \Omega \rightarrow \mathbb{R}$ are analytic functions. The set $E$ is called a subanalytic subset of $\mathcal{M}$ if every point $x \in \mathcal{M}$ has a neighbourhood $\Omega$ such that $E \cap \Omega$ is the image under the projection map $\mathcal{M} \times \mathbb{R}^{k} \rightarrow \mathcal{M}$ of a semi-analytic relatively compact subset of $\mathcal{M} \times \mathbb{R}^{k}$ (where $k$ depends on $x$ ). For the basic properties of semi-analytic and subanalytic sets see for instance [2], [11], [15], [22], [25].

For $A \subset \mathcal{M}$, we denote by $\varrho(\cdot, A)$ the distance function to $A$, i.e. $\varrho(z, A)=\inf _{x \in A} \varrho(z, x)$ if $A \neq \emptyset$, and $\varrho(z, \emptyset)=1$.

In the remainder of this section, $X, Y, Z$ are closed subanalytic sets in $\mathcal{M}$. We start with some remarks on $\mathcal{L}_{x_{0}}(X ; Y, Z)$.

REMARK 2.1. (a) If $x_{0} \in X \cap Y \subset Z$ and $X \cap \Omega \subset Z$ for some neighbourhood $\Omega$ of $x_{0}$, then obviously, for any $C, \nu>0$ the inequality (\#) holds in $\Omega$. So, $\mathcal{L}_{x_{0}}(X ; Y, Z)=0$. In order to omit this trivial case, we will assume $x_{0} \in \overline{X \backslash Z}$.

(b) Obviously, $\mathcal{L}_{x_{0}}(X, Y)=\mathcal{L}_{x_{0}}(Y, X)$. However, we cannot require that $\mathcal{L}_{x_{0}}(X ; Y, Z)=\mathcal{L}_{x_{0}}(Y ; X, Z)$. Indeed, for $X=\left\{\left(x_{1}, x_{2}\right): x_{2}=0\right\}$ and $Y=Z=\left\{\left(x_{1}, x_{2}\right): x_{1}=x_{2}\right\}$ we easily obtain $\mathcal{L}_{0}(X ; Y, Z)=1$ and $\mathcal{L}_{0}(Y ; X, Z)=0$.

Since the exponent $\mathcal{L}_{x_{0}}(X ; Y, Z)$ has a local character, the proof of Theorem 1.5 can be carried out in the case of subanalytic sets in an open set $G$ of a finite-dimensional real linear space $M$. This exponent does not depend on the choice of the norm, so we will use the Euclidean norm $|\cdot|$. Set $B\left(x_{0}, R\right)=\left\{x \in M:\left|x-x_{0}\right|<R\right\}$, where $x_{0} \in M$ and $R>0$.

LEMma 2.2. Let $Z$ be a closed subanalytic subset of $G$ and $x_{0} \in Z$. Let $R>0$ be such that $B=\overline{B\left(x_{0}, R\right)} \subset G$. Then

$$
A=\{(x, z) \in B \times(Z \cap B): \varrho(x, Z \cap B)=|x-z|\}
$$

is a nonempty compact and subanalytic set in $G \times M$. Moreover, if $Z$ is a semi-algebraic (and closed) subset of $M$, then the set $\{(x, z) \in M \times Z$ : $\varrho(x, Z)=|x-z|\}$ is semi-algebraic.

Proof. Since $\{(x, z, w) \in B \times(Z \cap B) \times(Z \cap B):|x-z|>|x-w|\}$ is a subanalytic and relatively compact subset of $G \times M^{2}$, its projection

$$
E=\left\{(x, z) \in B \times(Z \cap B): \exists_{w \in Z \cap B}|x-z|>|x-w|\right\}
$$

onto $B \times M$ is subanalytic. Moreover, $A=[B \times(Z \cap B)] \backslash E$, and hence, by the Gabrielov Theorem on Complement ([13, Theorem 1], [25, IV.4]), the set $A$ is subanalytic. The proof is analogous when $Z$ is semi-algebraic (by using the Tarski-Seidenberg Theorem, see [1, Theorem 2.3.4]). 
A curve $\varphi:[0, r) \rightarrow \mathcal{M}$, where $r>0$, is called analytic if $\varphi$ has an analytic extension $\psi:\left(r^{\prime}, r\right) \rightarrow \mathcal{M}$, where $r^{\prime}<0$. If $\mathcal{M}=M$, then in a neighbourhood of $0, \varphi$ is the sum of a power series of the form

$$
\varphi(t)=\alpha_{p} t^{p}+\alpha_{p+1} t^{p+1}+\cdots, \quad \alpha_{i} \in M, p \in \mathbb{Z}, p \geq 0 .
$$

If $\varphi \neq 0$, then we may assume that $\alpha_{p} \neq 0$. The number $p$ is called the order of $\varphi$ and denoted by ord $\varphi$. Additionally we put ord $0=\infty$.

Lemma 2.3. Let $x_{0} \in X \cap Y \subset Z$, where $x_{0} \in \overline{X \backslash Z}$, and let $B=$ $\overline{B\left(x_{0}, R\right)}$, where $R>0$ and $\overline{B\left(x_{0}, 2 R\right)} \subset G$. Then there exist analytic curves $\varphi:[0, r) \rightarrow X \cap B, \varphi_{1}:[0, r) \rightarrow Y$, and $\psi:[0, r) \rightarrow Z$, where $r>0$, such that

(i) $\varphi((0, r)) \subset X \backslash Z$,

(ii) $\varphi(0)=\varphi_{1}(0)=\psi(0)$,

(iii) there exists $C>0$ such that

where

$$
\varrho(x, Y) \geq C \varrho(x, Z)^{\nu} \quad \text { for } x \in B \cap X,
$$

$$
\nu=\frac{\operatorname{ord}\left(\varphi-\varphi_{1}\right)}{\operatorname{ord}(\varphi-\psi)}
$$

(iv) the smallest exponent $\nu$ for which (1) holds is defined by (2); moreover, there exist $C_{1}>0$ and $t_{0} \in(0, r)$ such that

$$
\varrho(\varphi(t), Y) \leq C_{1} \varrho(\varphi(t), Z)^{\nu} \quad \text { for } t \in\left[0, t_{0}\right] .
$$

Proof. For $x \in B$ and $E \subset G$ we have $\varrho(x, E)=\varrho\left(x, E \cap \overline{B\left(x_{0}, 2 R\right)}\right)$. Thus we may assume that $X \subset B$ and $Y, Z \subset \overline{B\left(x_{0}, 2 R\right)}$. Let

$$
\begin{aligned}
& V=\{(x, y, z) \in X \times Y \times Z: \varrho(x, Y)=|x-y| \wedge \varrho(x, Z)=|x-z|\}, \\
& U=\{((x, y, z),(a, b, c)) \in V \times V:|a-c|=|x-z| \wedge|x-y|>|a-b|\} .
\end{aligned}
$$

By Lemma 2.2, the sets $V$ and $U$ are subanalytic and relatively compact in $G \times M^{2}$ and $G \times M^{5}$, respectively. Then the projection $W=\{(x, y, z) \in V$ : $\left.\exists_{(a, b, c) \in V}((x, y, z),(a, b, c)) \in U\right\}$ of $U$ is a subanalytic set. So, the complement $\Gamma=V \backslash W$ is subanalytic. Obviously,

$\Gamma=\{(x, y, z) \in V: \varrho(x, Y)=\inf \{\varrho(a, Y): a \in X \cap B \wedge \varrho(a, Z)=\varrho(x, Z)\}\}$.

Since $B$ and $\overline{B\left(x_{0}, 2 R\right)}$ are compact sets, for any $a \in(X \cap B) \backslash Z$ there exists $(x, y, z) \in \Gamma$ such that $\varrho(x, Z)=\varrho(a, Z)$. By the assumption $x_{0} \in \overline{X \backslash Z}$, there exists $x_{1} \in X \cap Y$ such that $\left(x_{1}, x_{1}, x_{1}\right)$ is an accumulation point of $\Gamma$. Consequently, by the Curve Selection Lemma ([25, IV.3]), there exists an analytic curve $\left(\varphi, \varphi_{1}, \psi\right):[0, r) \rightarrow \Gamma$, where $r>0$, such that $\varphi, \varphi_{1}, \psi$ satisfy (i) and (ii).

For $\varphi, \varphi_{1}, \psi$ chosen above, let the number $\nu$ be given by (2). Obviously, $\nu \in \mathbb{Q}$ and $\nu>0$. By the definition of $\nu$, there exist $t_{0} \in(0, r)$ and $C_{1}, C_{2}>0$ 
such that

$$
\left|\varphi(t)-\varphi_{1}(t)\right| \leq C_{1}|\varphi(t)-\psi(t)|^{\nu} \leq C_{2}\left|\varphi(t)-\varphi_{1}(t)\right| \quad \text { for } t \in\left[0, t_{0}\right] .
$$

By the definition of $\Gamma$ we see that (4) implies (3).

Set $\varepsilon=\varrho\left(\varphi\left(t_{0}\right), Z\right)$; we have $\varepsilon>0$. Take any $x \in(X \backslash Z) \cap B$ such that $\varrho(x, Z)<\varepsilon$. Then there exists $t \in\left(0, t_{0}\right)$ such that $\varrho(x, Z)=\varrho(\varphi(t), Z)$. So, from (3) and the definition of $\Gamma$,

$$
\varrho(x, Y) \geq \varrho(\varphi(t), Y) \geq \frac{C_{1}}{C_{2}} \varrho(\varphi(t), Z)^{\nu}=\frac{C_{1}}{C_{2}} \varrho(x, Z)^{\nu} .
$$

Since $\{x \in X \cap B: \varrho(x, Z) \geq \varepsilon\}$ is compact, by (5), diminishing $C=C_{1} / C_{2}$ if necessary, we obtain (1) for $x \in X \cap B$. This gives (iii). The remaining condition in (iv) immediately follows from (3) and (4), because (4) holds only for $\nu$ given by (2).

By a stratification of a subset $X \subset \mathcal{M}$ we mean a decomposition of $X$ into a disjoint locally finite union

$$
X=\bigcup S_{\alpha},
$$

where the subsets $S_{\alpha}$ are called strata, such that each $S_{\alpha}$ is a connected embedded submanifold of $\mathcal{M}$, and each $\left(\bar{S}_{\alpha} \backslash S_{\alpha}\right) \cap X$ is the union of some strata of dimension smaller than $\operatorname{dim} S_{\alpha}$.

The set $X$ with stratification (6) is called locally bi-Lipschitz trivial along each stratum if for each stratum $S_{\alpha}$ and each $x \in S_{\alpha}$ there exist: a neighbourhood $U \subset \mathcal{M}$ of $x$, a submanifold $\mathcal{N}$ of $U$ transverse to $S_{\alpha}$ at $x$ and of dimension complementary to $\operatorname{dim} S_{\alpha}$, and a bi-Lipschitz homeomorphism (i.e. Lipschitz homeomorphism with Lipschitz inverse)

$$
\Psi: X \cap U \rightarrow\left(S_{\alpha} \cap U\right) \times(\mathcal{N} \cap X) .
$$

In [28] and [29] Parusiński showed the existence of a Lipschitz stratification of subanalytic sets, and proved that any Lipschitz stratification of $X$ ensures locally bi-Lipschitz triviality of $X$ along each stratum ([28, Theorem 1.9], and [29, Lipschitz Isotopy Lemma, Theorem 1.6]). From these results we obtain:

Lemma 2.4. Let $X=X_{1} \cup \cdots \cup X_{k}$, where $X_{1}, \ldots, X_{k}$ are compact subanalytic subsets of $M$. Then there exists a stratification $X=\bigcup S_{\alpha}$ of $X$ such that each $X_{1}, \ldots, X_{k}$ is a union of some strata $S_{\alpha}$, and $X$ is locally bi-Lipschitz trivial along each stratum. In particular, for each stratum $S_{\alpha}$ and any $y, z \in S_{\alpha}$ there exist neighbourhoods $\Omega_{y}, \Omega_{z}$ of $y, z$, respectively, and a bi-Lipschitz homeomorphism $\Phi: X \cap \Omega_{y} \rightarrow X \cap \Omega_{z}$ which preserves $X_{1}, \ldots, X_{k}$, i.e.

$$
\Phi\left(X_{i} \cap \Omega_{y}\right)=X_{i} \cap \Omega_{z} \quad \text { for } i=1, \ldots, k .
$$


Proof. By Theorems 1.4 and 1.6 in [29] there exists a stratification $X=$ $\bigcup S_{\alpha}$ of $X$ such that each $X_{1}, \ldots, X_{k}$ is the union of some strata $S_{\alpha}$, and $X$ is locally bi-Lipschitz trivial along each stratum $S_{\alpha}$. Take any stratum $S_{\alpha}$ which contains at least two points. Let $x \in S_{\alpha}$ and let $\Psi$ be a bi-Lipschitz homeomorphism of the form (7). One can assume that $\Psi$ is defined by a Lipschitz flow obtained by integrating a Lipschitz vector field tangent to strata of $X$ (see proof of Theorem 1.6 in [29] and proof of Proposition 1.1 in [26]). Then $\Psi\left(X_{i} \cap U\right)=\left(S_{\alpha} \cap U\right) \times\left(\mathcal{N} \cap X_{i}\right)$ for $i=1, \ldots, k$. Thus, for any $y \in S_{\alpha} \cap U$ we easily get (8). Since $S_{\alpha}$ is connected, we obtain the assertion.

Proof of Theorem 1.5. Without loss of generality we may assume that $x_{0} \in \overline{X \backslash Z}$. By Lemma 2.4, one can assume that there exists a stratification $X \cup Y \cup Z=\bigcup_{\alpha} S_{\alpha}$ such that each of the sets $X \cap Y, X, Y, Z$ is a union of some strata $S_{\alpha}$, and $X \cup Y \cup Z$ is locally bi-Lipschitz trivial along each stratum.

Take any $x_{0} \in X \cap Y$ and let $S_{\alpha_{1}}, \ldots, S_{\alpha_{k}}$ be all the strata for which $x_{0} \in \bar{S}_{\alpha}$. Let $R>0$ be such that $\overline{B\left(x_{0}, 2 R\right)} \subset G$ and

$$
(X \cup Y \cup Z) \cap \overline{B\left(x_{0}, 2 R\right)}=\left(S_{\alpha_{1}} \cup \cdots \cup S_{\alpha_{k}}\right) \cap \overline{B\left(x_{0}, 2 R\right)} .
$$

Let $\varphi:[0, r) \rightarrow X \cap \overline{B\left(x_{0}, R\right)}$ be an analytic curve for which there exist analytic curves $\varphi_{1}, \psi$ such that the assertion of Lemma 2.3 holds. Let $\nu$ be as in (2). Then $\nu$ is a rational number and satisfies (1). Hence, it suffices to prove that

$$
\nu=\mathcal{L}_{x_{0}}(X ; Y, Z) .
$$

In accordance with (1), it suffices to prove that for any $0<R_{1}<R$ there exists a continuous curve $\kappa:[0, \varepsilon) \rightarrow X \cap B_{1}$, where $\varepsilon>0$ and $B_{1}=$ $\overline{B\left(x_{0}, R_{1}\right)}$, such that $\kappa((0, \varepsilon)) \subset X \backslash Z, \kappa(0) \in X \cap Y$, and for some $C_{1}, C_{2}>0$,

$$
C_{1} \varrho(\kappa(t), Y) \leq \varrho(\kappa(t), Z)^{\nu} \leq C_{2} \varrho(\kappa(t), Y) \quad \text { for } t \in[0, \varepsilon) .
$$

Take any $0<R_{1}<R$. Let $x=\varphi(0)$, and let $x \in S_{\alpha_{i}}$. Then $S_{\alpha_{i}} \subset X \cap Y$ and there exists $y_{0} \in S_{\alpha_{i}}$ such that $\left|y_{0}-x_{0}\right|<R_{1} / 2$. By Lemma 2.4, there exist neighbourhoods $\Omega_{1}, \Omega_{2}$ of $x$ and $y_{0}$, respectively, where $\Omega_{2} \subset B_{1}$, and a bi-Lipschitz homeomorphism $\Phi:(X \cup Y \cup Z) \cap \Omega_{1} \rightarrow(X \cup Y \cup Z) \cap \Omega_{2}$ such that $\Phi\left(X \cap \Omega_{1}\right)=X \cap \Omega_{2}$ and $\Phi\left(Y \cap \Omega_{1}\right)=Y \cap \Omega_{2}$ and $\Phi\left(Z \cap \Omega_{1}\right)=$ $Z \cap \Omega_{2}$. Moreover, there exists $0<\varepsilon<t_{0}$ such that $\varphi([0, \varepsilon)) \subset \Omega_{1}$. Put $\kappa(t)=\Phi(\varphi(t))$ for $t \in[0, \varepsilon)$. Since $\Phi$ is a bi-Lipschitz homeomorphism,

$$
\begin{aligned}
& D_{1} \varrho(\varphi(t), Y) \leq \varrho(\kappa(t), Y) \leq D_{2} \varrho(\varphi(t), Y), \\
& D_{1} \varrho(\varphi(t), Z) \leq \varrho(\kappa(t), Z) \leq D_{2} \varrho(\varphi(t), Z)
\end{aligned}
$$

for $t \in[0, \varepsilon)$ and some $D_{1}, D_{2}>0$. Then by (3) we obtain (10) and, as a consequence, (9). This gives (i). Assertion (ii) follows from the above and Lemma 2.3 . 
EXAMPLE 2.5. In Theorems 1.4 and 1.5(ii), we cannot require that $\varphi(0)$ $=x_{0}$. Indeed, let $x_{0}=0 \in \mathbb{R}^{3}$ and

$$
X=\left\{\left(x_{1}, x_{2}, x_{3}\right) \in \mathbb{R}^{3}: x_{1}^{2}=x_{2} x_{3}\right\}, \quad Y=\left\{\left(x_{1}, x_{2}, x_{3}\right) \in \mathbb{R}^{3}: x_{2}=0\right\} .
$$

Then $X \cap Y=\left\{\left(x_{1}, x_{2}, x_{3}\right) \in \mathbb{R}^{3}: x_{1}=x_{2}=0\right\}$. Let $Z=X \cap Y$.

By Theorem 1.1, $\mathcal{L}_{0}(X, Y)=\mathcal{L}_{0}(X ; Y, Z)$. Note that $\mathcal{L}_{0}(X ; Y, Z)=2$. Indeed, we may use the polycylindric norm in $\mathbb{R}^{3}$. Let $\Omega=\left\{\left(x_{1}, x_{2}, x_{3}\right) \in\right.$ $\left.\mathbb{R}^{3}: \max \left\{\left|x_{1}\right|,\left|x_{2}\right|,\left|x_{3}\right|\right\}<\varepsilon\right\}, 0<\varepsilon<1$. For any $x=\left(x_{1}, x_{2}, x_{3}\right) \in X \cap \Omega$ we have $\varrho(x, Y)=\left|x_{2}\right|$ and $\varrho(x, Z)=\max \left\{\left|x_{1}\right|,\left|x_{2}\right|\right\}$. If $\varrho(x, Z)=\left|x_{2}\right|$, then

$$
\varrho(x, Y)=\left|x_{2}\right| \geq\left|x_{2}\right|^{2}=\varrho(x, Z)^{2} .
$$

If $\varrho(x, Z)=\left|x_{1}\right|$, then

$$
\varrho(x, Y)=\left|x_{2}\right| \geq\left|x_{2} x_{3}\right|=\left|x_{1}\right|^{2}=\varrho(x, Z)^{2} .
$$

So, $\mathcal{L}_{0}(X ; Y, Z) \leq 2$. On the other hand, taking the curve $\varphi:[0, \varepsilon / 2) \ni t \mapsto$ $\left(t,(2 / \varepsilon) t^{2}, \varepsilon / 2\right) \in X \cap \Omega$, we have

$$
\varrho(\varphi(t), Y)=\frac{2}{\varepsilon} t^{2}=\frac{2}{\varepsilon} \varrho(\varphi(t), Z)^{2} .
$$

Hence $\mathcal{L}_{0}(X ; Y, Z) \geq 2$. Summing up, $\mathcal{L}_{0}(X, Y)=\mathcal{L}_{0}(X ; Y, Z)=2$.

We shall show that the exponent $\mathcal{L}_{0}(X, Y)$ is not attained on any analytic curve $\varphi$ such that $\varphi(0)=0$. Assume to the contrary that for some analytic curve $\varphi=\left(\varphi_{1}, \varphi_{2}, \varphi_{3}\right):[0, r) \rightarrow \mathbb{R}^{3}$, where $\varphi(0)=0, \varphi((0, r)) \subset \mathbb{R}^{3} \backslash Z$, we have $\varrho(\varphi(t), X)+\varrho(\varphi(t), Y) \leq C \varrho(\varphi(t), Z)^{2}$ for $t \in[0, r)$, where $C>0$. Then

$$
\varrho(\varphi(t), X) \leq C \varrho(\varphi(t), Z)^{2}, \quad \varrho(\varphi(t), Y) \leq C \varrho(\varphi(t), Z)^{2} \quad \text { for } t \in[0, r) .
$$

Since $\varrho(\varphi(t), Y)=\left|\varphi_{2}(t)\right|, \varrho(\varphi(t), Z)=\max \left\{\left|\varphi_{1}(t)\right|,\left|\varphi_{2}(t)\right|\right\}$ for $t \in[0, r)$, from (11) we may assume $\varrho(\varphi(t), Z)=\left|\varphi_{1}(t)\right|$ for $t \in[0, r)$. So, (11) gives

$$
0<\operatorname{ord} \varphi_{1}<\infty \quad \text { and } \operatorname{ord} \varphi_{2} \geq 2 \operatorname{ord} \varphi_{1} .
$$

By (11), the origin is an accumulation point of the subanalytic set

$$
\begin{aligned}
& E=\left\{\left(x_{1}, x_{2}, x_{3}, y_{1}, y_{2}, y_{3}\right) \in \mathbb{R}^{6}: x_{1} \neq 0 \wedge y_{1}^{2}=y_{2} y_{3}\right. \\
&\left.\wedge \max \left\{\left|x_{2}\right|,\left|x_{1}-y_{1}\right|,\left|x_{2}-y_{2}\right|,\left|x_{3}-y_{3}\right|\right\} \leq C\left|x_{1}\right|^{2}\right\} .
\end{aligned}
$$

So, by the Curve Selection Lemma we may assume that there exists an analytic curve $\psi=\left(\psi_{1}, \psi_{2}, \psi_{3}\right):[0, r) \rightarrow X$ such that $\psi(0)=0$ and $(\varphi(t), \psi(t)) \in E$ for $t \in(0, r)$. Then

$$
\left|\varphi_{i}(t)-\psi_{i}(t)\right| \leq C\left|\varphi_{1}(t)\right|^{2} \quad \text { for } t \in[0, r),
$$

and so $\operatorname{ord}\left(\varphi_{i}-\psi_{i}\right) \geq 2 \operatorname{ord} \varphi_{1}, i=1$, 2 Since $0<\operatorname{ord} \varphi_{1}<2 \operatorname{ord} \varphi_{1}$, wee have ord $\psi_{1}=\operatorname{ord} \varphi_{1}$. Moreover, $\psi_{1}^{2}=\psi_{2} \psi_{3}$ and $\psi(0)=0$, hence $\operatorname{ord} \psi_{2}<2 \operatorname{ord} \psi_{1}$. Therefore, ord $\varphi_{2}=$ ord $\psi_{2}<\infty$. Hence and from (12),

$$
\operatorname{ord} \varphi_{2} \geq 2 \operatorname{ord} \varphi_{1}=2 \operatorname{ord} \psi_{1}>\operatorname{ord} \psi_{2}=\operatorname{ord} \varphi_{2}
$$

This is impossible. 
Corollary 2.6. Let $X, Y, Z$ be compact subanalytic subsets of a finitedimensional real linear space $M$ such that $X \cap Y \subset Z$. Then there exists a stratification

$$
X \cap Y=\bigcup S_{\alpha}
$$

such that for each stratum $S_{\alpha}$, the function

$$
S_{\alpha} \ni x \mapsto \mathcal{L}_{x}(X ; Y, Z)
$$

is constant. In particular, the function $X \cap Y \ni x \mapsto \mathcal{L}_{x}(X ; Y, Z)$ is upper semi-continuous.

Proof. By Lemma 2.4, one can assume that there exists a stratification $X \cup Y \cup Z=\bigcup_{\alpha} S_{\alpha}$ such that each of $X \cap Y, X, Y, Z$ is a union of some strata $S_{\alpha}$, and $X \cup Y \cup Z$ is locally bi-Lipschitz trivial along each stratum.

Let $S_{\alpha} \subset X \cap Y$. Take any $z, w \in S_{\alpha}$. Then there exist neighbourhoods $\Omega_{1}$, $\Omega_{2}$ of $z, w$ respectively and a bi-Lipschitz homeomorphism $\Phi:(X \cup Y \cup Z)$ $\cap \Omega_{1} \rightarrow(X \cup Y \cup Z) \cap \Omega_{2}$ such that $\Phi\left(X \cap \Omega_{1}\right)=X \cap \Omega_{2}, \Phi\left(Y \cap \Omega_{1}\right)=Y \cap \Omega_{2}$ and $\Phi\left(Z \cap \Omega_{1}\right)=Z \cap \Omega_{2}$. If $X \cap \Omega \subset Z$ for some neighbourhood $\Omega$ of $z$, then $X \cap \Phi\left(\Omega \cap \Omega_{1}\right) \subset Z$, so $\mathcal{L}_{z}(X ; Y, Z)=\mathcal{L}_{w}(X ; Y, Z)=0$. Assume that $z, w \in \overline{X \backslash Z}$. By Theorem 1.5, one can assume that

$$
\begin{array}{ll}
\varrho(x, Y) \geq C_{1} \varrho(x, Z)^{\mathcal{L}_{z}(X ; Y, Z)} & \text { for } x \in X \cap \Omega_{1}, \\
\varrho(x, Y) \geq C_{2} \varrho(x, Z)^{\mathcal{L}_{w}(X ; Y, Z)} & \text { for } x \in X \cap \Omega_{2},
\end{array}
$$

where $C_{1}, C_{2}>0$ are some constants. Since $\Phi$ is a bi-Lipschitz homeomorphism, we have $\mathcal{L}_{z}(X ; Y, Z)=\mathcal{L}_{w}(X ; Y, Z)$, and so the function (14) is constant.

Corollary 2.7. For any closed semi-algebraic subsets $X, Y, Z$ of a linear space $M$ such that $X \cap Y \subset Z$, the set $\left\{\mathcal{L}_{x}(X ; Y, Z): x \in X \cap Y\right\}$ is finite.

Proof. Let $B=\{z \in M:|z|<1\}$. The mapping

$$
H: B \ni z \mapsto \frac{z}{1-|z|^{2}} \in M
$$

is a diffeomorphism. The inverse of $H$ is of the form

$$
H^{-1}(w)=w \frac{2}{1+\sqrt{1+4|w|^{2}}} .
$$

Moreover, $H$ and $H^{-1}$ are semi-algebraic and locally bi-Lipschitz homeomorphisms. Let $E=H^{-1}(X), W=H^{-1}(Y), V=H^{-1}(Z)$. Then $E \cup W \cup V$ is a semi-algebraic set, and in consequence, $A=\bar{E} \cup \bar{W} \cup \bar{V}$ is a compact semialgebraic set. By Corollary 2.6, there exists a stratification $\bar{E} \cap \bar{W}=\bigcup S_{\alpha}$ such that for each stratum $S_{\alpha}$ the function $S_{\alpha} \cap B \ni z \mapsto \mathcal{L}_{z}(E ; W, V)$ is constant, and the number of strata $S_{\alpha}$ is finite. Since $X=H(E), Y=H(W)$, 
$Z=H(V), X \cap Y=H(E \cap W)=\bigcup H\left(S_{\alpha} \cap B\right)$ and $H$ is locally biLipschitz homeomorphism, it follows that for any $H\left(S_{\alpha} \cap B\right) \neq \emptyset$ the mapping $H\left(S_{\alpha} \cap B\right) \ni x \mapsto \mathcal{L}_{x}(X ; Y, Z)$ is constant. This gives the assertion.

By the $i$-th skeleton of the stratification (6) we mean $X^{i}=\bigcup_{\operatorname{dim} S_{\alpha} \leq i} S_{\alpha}$. The stratification (6) of a complex analytic subset $X$ of a complex analytic manifold $\mathcal{M}$ is called complex analytic if all the skeletons $X^{i}$ are complex analytic subsets of $\mathcal{M}$. The stratification (6) of a complex algebraic subset $X$ of a complex linear space $M$ is called complex algebraic if all the skeletons $X^{i}$ are complex algebraic subsets of $M$ and the number of strata $S_{\alpha}$ is finite.

REMARK 2.8. Parusiński (in [28, Theorems 2.4 and 2.6]) proved that for a complex analytic set $X$, we may require that the locally Lipschitz stratification (6) is complex analytic, and for a complex algebraic set, we may require that the stratification is complex algebraic. Hence, in Corollary 2.6, for complex analytic sets $X, Y, Z$, we may require that the stratification (13) is complex analytic, and for complex algebraic sets $X, Y, Z$, we may require that it is complex algebraic.

REMARK 2.9. Let $X, Y, Z$ be closed subanalytic subsets of an open subset $G \subset \mathbb{R}^{n}$ such that $X \cap Y \subset Z$. Under a given locally Lipschitz stratification

$$
X \cup Y \cup Z=\bigcup S_{\alpha}
$$

such that $X \cap Y$ is a union of some strata $S_{\alpha}$, for any $x_{0} \in X \cap Y$ such that $x_{0} \in \overline{X \backslash Z}$, we may determine a neighbourhood $\Omega$ of $x_{0}$ on which the inequality (\#) holds for $\nu=\mathcal{L}_{x_{0}}(X ; Y, Z)$.

Indeed, let $S_{\alpha_{1}}, \ldots, S_{\alpha_{k}}$ be all the strata for which $x_{0} \in \bar{S}_{\alpha}$. Take any $R>0$ such that $B=\overline{B\left(x_{0}, R\right)} \subset G$ and $(X \cup Y \cup Z) \cap B=\left(S_{\alpha_{1}} \cup \cdots \cup S_{\alpha_{k}}\right) \cap B$. Put $\Omega=\left\{x \in \mathbb{R}^{n}:\left|x-x_{0}\right|<R / 2\right\}$. Under the notation of the proof of Theorem 1.5, we obtain (1) for $\nu=\mathcal{L}_{x_{0}}(X ; Y, Z)$. For $x \in X$ such that $\left|x-x_{0}\right|<R / 2$, we have $\varrho(x, Y)=\varrho(x, Y \cap B)$ and $\varrho(x, Z)=\varrho(x, Z \cap B)$. Thus, by (1), we obtain $\varrho(x, Y) \geq C \varrho(x, Z)^{\mathcal{L}_{x_{0}}(X ; Y, Z)}$ for $x \in X \cap \Omega$.

3. Łojasiewicz exponent of a mapping. Let $X, Y$ be closed subanalytic subsets of $\mathcal{M}$, and let $F: X \rightarrow Y$ be a subanalytic mapping, i.e. a continuous mapping with subanalytic graph $\Gamma(F)$.

From Theorem 1.5 we get (cf. [3, Corollary 1], [20, Corollary 6.4]):

Corollary 3.1. Let $x_{0} \in X, y_{0}=F\left(x_{0}\right)$, and $V=F^{-1}\left(y_{0}\right)$. The number $\mathcal{L}_{x_{0}}(F)=\mathcal{L}_{\left(x_{0}, y_{0}\right)}\left(\Gamma(F) ; X \times\left\{y_{0}\right\}, V \times Y\right)$ is the smallest exponent $\nu$ satisfying (€) for some $C>0$ and a neighbourhood $\Omega$ of $x_{0}$.

Proof. For any $x \in X$ we have $\varrho\left(F(x), y_{0}\right)=\varrho\left((x, F(x)), X \times\left\{y_{0}\right\}\right)$ and $\varrho(x, V)=\varrho((x, F(x)), V \times Y)$. So, condition (E) is equivalent to the 
inequality $\varrho\left(z, X \times\left\{y_{0}\right\}\right) \geq C \varrho(z, V \times Y)^{\nu}, z \in \Gamma(F) \cap \Delta$, where $\Delta$ is a neighbourhood of $\left(x_{0}, y_{0}\right)$. Thus, Theorem 1.5 gives the assertion.

The number $\mathcal{L}_{x_{0}}(F)$ is called the Eojasiewicz exponent of $F$ at $x_{0}$. From Corollaries 3.1 and 2.6, we immediately obtain

Corollary 3.2. Let $V=F^{-1}\left(y_{0}\right)$, where $y_{0} \in Y$. The function $V \ni$ $x \mapsto \mathcal{L}_{x}(F)$ is upper semi-continuous.

Remark 3.3. Let $V=F^{-1}\left(F\left(x_{0}\right)\right)$ and let $x_{0} \in \overline{X \backslash V}$. By Corollary 3.1 and Theorem 1.5, the exponent $\mathcal{L}_{x_{0}}(F)$ is attained on an analytic curve, i.e. for any neighbourhood $\Omega$ of $x_{0}$ there exist $C_{1}>0$ and an analytic curve $\varphi:[0, r) \rightarrow X \cap \Omega$ such that $\varphi(0) \in V, \varphi((0, r)) \subset X \backslash V$ and $\varrho\left(F(\varphi(t)), y_{0}\right) \leq C_{1} \varrho(\varphi(t), V)^{\mathcal{L}_{x_{0}}(F)}$ for $t \in[0, r)$. We cannot require that $\varphi(0)=x_{0}$. Indeed, it suffices to consider the set $X$ from Example 2.5 and the projection map $F\left(x_{1}, x_{2}, x_{3}\right)=x_{2}$.

In the remainder of this section, $F: X \rightarrow \mathbb{R}^{m}$ is a semi-algebraic mapping, i.e. a continuous mapping defined on a semi-algebraic set $X \subset \mathbb{R}^{n}$ with semialgebraic graph $\Gamma(F)$. We assume that $X$ is closed. Let $V=F^{-1}(0)$.

According to Corollaries 2.7 and 3.1, the set $\left\{\mathcal{L}_{x}(F): x \in V\right\}$ is finite, so we may define a special regular separation exponent of $F$,

$$
\mathcal{L}(F)=\max \left\{\mathcal{L}_{x}(F): x \in V\right\} \quad \text { if } V \neq \emptyset .
$$

Additionally, we put $\mathcal{L}(F)=0$ if $V=\emptyset$.

REMARK 3.4. (a) Obviously, $\mathcal{L}(F)=0$ if and only if for each connected component $W$ of $X$ either $V \cap W=W$ or $V \cap W=\emptyset$.

(b) From the definition of $\mathcal{L}(F)$, it follows that if $X$ is a compact set, then there exists $C>0$ such that $|F(x)| \geq C \varrho(x, V)^{\mathcal{L}(F)}$ for $x \in X$.

In the considerations at infinity we will use the notion of curves meromorphic at infinity instead of analytic curves. A curve $\varphi:[a, \infty) \rightarrow \mathbb{R}^{k}$, where $a \in \mathbb{R}$, is called meromorphic at infinity if $\varphi$ is the sum of a Laurent series of the form

$$
\varphi(t)=\alpha_{p} t^{p}+\alpha_{p-1} t^{p-1}+\cdots, \quad \alpha_{i} \in \mathbb{R}^{k}, p \in \mathbb{Z} .
$$

If $\varphi \neq 0$, then we may assume that $\alpha_{p} \neq 0$. The number $p$ is called the degree of $\varphi$ and denoted by $\operatorname{deg} \varphi$. Additionally, we put $\operatorname{deg} 0=-\infty$.

TheOREM 3.5. If $X \backslash V$ is an unbounded set, then for any $\nu \in \mathbb{Q}$ such that $\nu \geq \mathcal{L}(F)$, there exists a unique $l \in \mathbb{Q}$ such that for some constant $C>0$,

$$
|F(x)|(1+|x|)^{l} \geq C \varrho(x, V)^{\nu} \quad \text { for } x \in X,
$$


and for some curve $\varphi:[a, \infty) \rightarrow X \backslash V$ meromorphic at infinity, with $\operatorname{deg} \varphi>0$,

$$
|F(\varphi(t))|(1+|\varphi(t)|)^{l} \leq C^{\prime} \varrho(\varphi(t), V)^{\nu}, \quad t \in[a, \infty),
$$

where $C^{\prime}>0$ is a constant.

Proof. For any $r>0$, the set $\{x \in X:|x| \leq r\}$ is compact, so there exists $C_{r}>0$ such that

$$
C_{r} \varrho(x, V)^{\nu} \leq|F(x)| \quad \text { for } x \in X,|x| \leq r .
$$

Observe that the set

$$
W=\left\{w \in X \backslash V: \forall_{x \in X}\left(|w|=|x| \Rightarrow 2 \varrho(w, V)^{\nu}|F(x)| \geq \varrho(x, V)^{\nu}|F(w)|\right)\right\}
$$

is unbounded. Indeed, since $X \backslash V$ is unbounded, for any sufficiently large $r>0$ the set

$$
A=\left\{\varrho(x, V)^{\nu} /|F(x)|:|x|=r \wedge x \in X \backslash V\right\}
$$

is nonempty, and $A \subset(0, \infty)$. Thus, from (17) we get $0<\sup A \leq 1 / C_{r}$, and therefore, there exists $w \in X \backslash V$ such that $|w|=r$ and

$$
\frac{\varrho(w, V)^{\nu}}{|F(w)|}>\frac{1}{2} \sup A \text {. }
$$

This implies that $w \in W$. In consequence, $W$ is an unbounded set.

Since $\nu$ is a rational number, by Lemma 2.2 and the Tarski-Seidenberg Theorem we conclude that $W$ is a semi-algebraic set. Moreover, $W$ is unbounded, thus, by the Curve Selection Lemma at infinity, there exists a curve $\left(\varphi, \varphi_{1}\right):[a, \infty) \rightarrow \Gamma\left(\left.F\right|_{W}\right)$ meromorphic at infinity such that $\operatorname{deg} \varphi>0$, $\varphi_{1}=F(\varphi), \operatorname{deg} \varphi_{1} \in \mathbb{Z}$. If $V \neq \emptyset$, then by Lemma 2.2 we may assume that $\varrho(\varphi(t), V)=|\varphi(t)-\psi(t)|$ for $t \in[a, \infty)$, where $\psi:[a, \infty) \rightarrow V$ is a curve meromorphic at infinity. If $V=\emptyset$, we put $\psi=\varphi+1$. Let

$$
l=\frac{\nu \operatorname{deg}(\varphi-\psi)-\operatorname{deg} F(\varphi)}{\operatorname{deg} \varphi} .
$$

Obviously $l \in \mathbb{Q}$. Moreover, there exist $C^{\prime}, C^{\prime \prime}>0$ and $R>0$, where $R=$ $\left|\varphi\left(t_{0}\right)\right|$ for some $t_{0}$, such that for any $t \in[a, \infty)$ satisfying $|\varphi(t)|>R$, we have

$$
2 C^{\prime \prime} \frac{\varrho(\varphi(t), V)^{\nu}}{|F(\varphi(t))|} \leq(1+|\varphi(t)|)^{l} \leq C^{\prime} \frac{\varrho(\varphi(t), V)^{\nu}}{|F(\varphi(t))|} .
$$

Take any $x \in X \backslash V$ such that $|x|>R$. Since $\operatorname{deg} \varphi>0$, we have $|x|=|\varphi(t)|$ for some $t \in[a, \infty)$. By the definition of $W$ and from (18),

$$
C^{\prime \prime} \frac{\varrho(x, V)^{\nu}}{|F(x)|} \leq 2 C^{\prime \prime} \frac{\varrho(\varphi(t), V)^{\nu}}{|F(\varphi(t))|} \leq(1+|\varphi(t)|)^{l}=(1+|x|)^{l} .
$$

Let $C=\min \left\{C^{\prime \prime}, C_{R} \min \left\{1,(1+R)^{l}\right\}\right\}$. Then (19) gives (15) for $x \in X$ such that $|x|>R$. Since $(1+|x|)^{l} \geq \min \left\{1,(1+R)^{l}\right\}$ for $|x| \leq R,(17)$ gives (15) 
for $x \in X$ such that $|x| \leq R$. Summing up, (15) holds in $X$. Moreover, (16) immediately follows from (18).

For any $\nu \in \mathbb{Q}$ such that $\nu \geq \mathcal{L}(F)$, we denote by $l_{\infty}(F, \nu)$ the unique number $l \in \mathbb{Q}$ satisfying (15) and (16) of the assertion of Theorem 3.5. If $\nu=\mathcal{L}(F)$, then for simplicity we write $l_{\infty}(F)$ instead of $l_{\infty}(F, \mathcal{L}(F))$.

REMARK 3.6. In the case when $V$ is finite, the Łojasiewicz exponent of $F$ at infinity $\mathcal{L}_{\infty}(F)$ has been investigated, where

$$
\mathcal{L}_{\infty}(F)=\sup \left\{\nu \in \mathbb{R}: \exists_{C, R>0} \forall_{x \in X}\left(|x| \geq R \Rightarrow|F(x)| \geq C|x|^{\nu}\right)\right\} .
$$

It is easy to see that, in this case, we have $\mathcal{L}_{\infty}(F)=\mathcal{L}(F)-l_{\infty}(F)$.

This exponent has been applied in many problems concerning polynomial mappings (see for instance [5]-[8], [14], [16], [18], [30], [31], [33], [34]).

In the case of polynomial mappings $F: \mathbb{R}^{n} \rightarrow \mathbb{R}^{m}$, estimations from above of $\mathcal{L}_{x_{0}}(F)$ are very interesting. In the complex case this has been done ([4], [9], [10], [17], [18]). The real case is more difficult. We have the following:

Proposition 3.7. Let $F=\left(f_{1}, \ldots, f_{m}\right): \mathbb{R}^{n} \rightarrow \mathbb{R}^{m}$ be a polynomial mapping, $F_{\mathbb{C}}: \mathbb{C}^{n} \rightarrow \mathbb{C}^{m}$ be the complexification of $F, V=F^{-1}(0)$ and $V_{\mathbb{C}}=F_{\mathbb{C}}^{-1}(0)$. Then, for any $x_{0} \in V$,

$$
\mathcal{L}_{x_{0}}(F) \leq \mathcal{L}_{x_{0}}\left(F_{\mathbb{C}}\right) \mathcal{L}_{x_{0}}\left(V_{\mathbb{C}}, \mathbb{R}^{n}\right) .
$$

Moreover, if $d=\max _{1 \leq j \leq m} \operatorname{deg} f_{j}>0$, then

$$
\mathcal{L}_{x_{0}}(F) \leq d \mathcal{L}_{x_{0}}\left(W_{\mathbb{C}}, \mathbb{R}^{n}\right),
$$

where $W_{\mathbb{C}} \subset \mathbb{C}^{n}$ is the zero-set of the complexification of $g=f_{1}^{2}+\cdots+f_{m}^{2}$.

Proof. The inequality (20) follows immediately from the definition.

It is easy to observe that $\mathcal{L}_{x_{0}}(F)=\frac{1}{2} \mathcal{L}_{x_{0}}(g)$. As the degree of $\Gamma\left(g_{\mathbb{C}}\right)$ is equal to $2 d$, by Theorem 4.2 in [10] we obtain $\mathcal{L}_{x_{0}}\left(g_{\mathbb{C}}\right) \leq 2 d$. Hence and from (20) we get (21).

EXAMPLE 3.8. For a polynomial function $g: \mathbb{R}^{n} \rightarrow \mathbb{R}$, and its complex zero-set $W_{\mathbb{C}}=g_{\mathbb{C}}^{-1}(0)$, the exponent $\mathcal{L}_{x_{0}}\left(W_{\mathbb{C}}, \mathbb{R}^{n}\right)$ for $x_{0} \in \mathbb{R}^{n} \cap W_{\mathbb{C}}$ can be large.

Indeed, we take the Masser and Philippon example ([17, Example 15]). Let $f_{1}(x)=x_{2}-x_{1}^{d}, f_{2}(x)=x_{3}-x_{2}^{d}, \ldots, f_{n-1}(x)=x_{n}-x_{n-1}^{d}, f_{n}(x)=x_{n}^{d}$, and $g=f_{1}^{2}+\cdots+f_{n}^{2}$, for $x=\left(x_{1}, \ldots, x_{n}\right)$. Let $F=\left(f_{1}, \ldots, f_{n}\right): \mathbb{R}^{n} \rightarrow \mathbb{R}^{n}$. Then $\mathcal{L}_{0}(F) \geq d^{n}$, and by $(21), d^{n} \leq d \mathcal{L}_{0}\left(W_{\mathbb{C}}, \mathbb{R}^{n}\right)$, i.e. $\mathcal{L}_{0}\left(W_{\mathbb{C}}, \mathbb{R}^{n}\right) \geq d^{n-1}$.

4. Remarks on separation of two mappings. Let $X, Y, Z$ be closed subanalytic sets and let $F: X \rightarrow Y$ and $g: X \rightarrow Z$ be subanalytic mappings, $x_{0} \in X, y_{0}=F\left(x_{0}\right), V=F^{-1}\left(y_{0}\right) \subset g^{-1}\left(z_{0}\right)$, where $z_{0} \in Z$. From Theorem 1.5 and Corollary 2.6, we easily obtain 
Corollary 4.1. $\mathcal{L}_{x_{0}}(F / g)=\mathcal{L}_{\left(x_{0}, y_{0}, z_{0}\right)}\left(\Gamma(F, g) ; X \times\left\{y_{0}\right\} \times Z, X \times Y\right.$ $\left.\times\left\{z_{0}\right\}\right)$ is the smallest exponent $\nu$ satisfying (LT) for some $C>0$ and a neighbourhood $\Omega$ of $x_{0}$. Moreover, the function $V \ni x \mapsto \mathcal{L}_{x}(F / g)$ is upper semi-continuous.

REMARK 4.2. If $g^{-1}\left(z_{0}\right) \cap \Omega=V \cap \Omega$ for some neighbourhood $\Omega$ of $x_{0}$, then $\mathcal{L}_{x_{0}}(F) \leq \mathcal{L}_{x_{0}}(F / g) \mathcal{L}_{x_{0}}(g)$.

Indeed, $\varrho_{\mathcal{N}}\left(F(x), y_{0}\right) \geq D \varrho_{\mathcal{N}}\left(g(x), z_{0}\right)^{\mathcal{L}_{x_{0}}(F / g)} \geq D^{\prime} \varrho(x, V)^{\mathcal{L}_{x_{0}}(F / g) \mathcal{L}_{x_{0}}(g)}$ in a neighbourhood of $x_{0}$ for some constants $D, D^{\prime}>0$.

REMARK 4.3. Let $F: \mathcal{M} \rightarrow \mathbb{R}^{k}$ and $g: \mathcal{M} \rightarrow \mathbb{R}^{m}$ be analytic mappings, $V=F^{-1}(0) \subset g^{-1}(0)$, and let $x_{0} \in V$.

(a) We have $\mathcal{L}_{x_{0}}(F) \geq \mathcal{L}_{x_{0}}(F / g)$. Indeed, $g$ is a locally Lipschitz mapping, so $|g(x)|^{\mathcal{L}_{x_{0}}(F)} \leq C \varrho\left(x, g^{-1}(0)\right)^{\mathcal{L}_{x_{0}}(F)} \leq C \varrho(x, V)^{\mathcal{L}_{x_{0}}(F)} \leq C^{\prime}|F(x)|$ in a neighbourhood of $x_{0}$ for some $C, C^{\prime}>0$.

(b) If $x_{0}$ is a smooth point of $V$, then $\mathcal{L}_{x_{0}}(F / g)=\mathcal{L}_{x_{0}}(F)$, provided the components of $g$ generate the ideal of the germ of $V$ at $x_{0}$. In particular $\mathcal{L}_{x_{0}}(F)=\sup _{h} \mathcal{L}_{x_{0}}(F / h)$, where $h$ runs through all analytic mappings such that $V \cap \Omega \subset h^{-1}(0)$ for some neighbourhood $\Omega$ of $x_{0}$. Indeed, it is easy to see that $\mathcal{L}_{x_{0}}(g)=1$, and then (a) and Remark 4.2 give the assertion.

(c) If $x_{0}$ is a singular point of $V$, then we can require neither $\mathcal{L}_{x_{0}}(F)=$ $\sup _{h} \mathcal{L}_{x_{0}}(F / h)$ nor $\mathcal{L}_{x_{0}}(F)>\sup _{h} \mathcal{L}_{x_{0}}(F / h)$.

Indeed, for $F(x, y)=x y,(x, y) \in \mathbb{R}^{2}$, we have $\mathcal{L}_{0}(F)=2$. Moreover, for any nonzero analytic mapping $h$ such that $h(x, y)=0$ for $x y=0$ in a neighbourhood of 0 , we have $|h(x, y)|=|F(x, y)|\left|h_{1}(x, y)\right|$, where $h_{1}$ is an analytic mapping. Thus, $\mathcal{L}_{0}(F / h) \leq 1<2=\mathcal{L}_{0}(F)$.

On the other hand, for $F(x, y, z)=\left(x^{2}, y z\right)$ and $g(x, y, z)=(x, y z)$, we have $V=F^{-1}(0,0)=(\{0\} \times \mathbb{R} \times\{0\}) \cup(\{0,0\} \times \mathbb{R})$, and in the polycylindric norm, $\varrho((x, y, z), V)=\min \{\max \{|x|,|y|\}, \max \{|x|,|z|\}\}$. Then we easily deduce that $\mathcal{L}_{0}(F)=2=\mathcal{L}_{0}(F / g)$.

Let $F: X \rightarrow \mathbb{R}^{k}$ and $g: X \rightarrow \mathbb{R}^{m}$ be semi-algebraic mappings, $V=$ $F^{-1}(0)$, and let $V \subset g^{-1}(0)$. By Corollaries 2.7 and 4.1 , the set $\left\{\mathcal{L}_{x}(F / g)\right.$ : $x \in V\}$ is finite. Then we may define

$$
\mathcal{L}(F / g)=\max \left\{\mathcal{L}_{x}(F / g): x \in V\right\} \quad \text { if } V \neq \emptyset .
$$

Additionally we put $\mathcal{L}(F / g)=0$ if $V=\emptyset$.

REMARK 4.4. (a) Obviously, $\mathcal{L}(F / g)=0$ if and only if for each connected component $W$ of $X$ either $W \subset g^{-1}(0)$ or $V \cap W=\emptyset$.

(b) From the definition of $\mathcal{L}(F / g)$ it follows that if $X$ is a compact set, then there exists $C>0$ such that $|F(x)| \geq C|g(x)|^{\mathcal{L}(F / g)}$ for $x \in X$.

Repeating the proof of Theorem 3.5 (by considering $|g(x)|$ instead of $\varrho(x, V))$ we obtain 
THeOREM 4.5. If $X \backslash V$ is an unbounded set, then for any $\nu \in \mathbb{Q}$ such that $\nu \geq \mathcal{L}(F / g)$, there exists a unique $l \in \mathbb{Q}$ such that for some constant $C>0$,

$$
|F(x)|(1+|x|)^{l} \geq C|g(x)|^{\nu} \quad \text { for any } z \in X,
$$

and for some curve $\varphi:[r, \infty) \rightarrow X \backslash V$ meromorphic at infinity, with $\operatorname{deg} \varphi>0$,

$$
|F(\varphi(t))|(1+|\varphi(t)|)^{l} \leq C^{\prime}|g(\varphi(t))|^{\nu}, \quad t \in[r, \infty),
$$

where $C^{\prime}>0$ is a constant.

For any $\nu \in \mathbb{Q}$ such that $\nu \geq \mathcal{L}(F / g)$, the unique number $l \in \mathbb{Q}$ satisfying the assertion of Theorem 4.5 is denoted by $l_{\infty}(F / g, \nu)$. If $\nu=\mathcal{L}(F / g)$, then, for simplicity, we write $l_{\infty}(F / g)$.

In the case of polynomial mappings $F: \mathbb{R}^{n} \rightarrow \mathbb{R}^{k}$ and $g: \mathbb{R}^{n} \rightarrow \mathbb{R}^{m}$, we have the following connection between $l_{\infty}(F / g, \nu), l_{\infty}(F, \nu)$ and $\mathcal{L}(F)$.

COROLlary 4.6. Let $g_{1}, \ldots, g_{m}$ be the components of $g$, and $d=$ $\max _{1 \leq j \leq m} \operatorname{deg} g_{j}$. If $V \neq \emptyset$ and $d>0$, then

$$
l_{\infty}(F / g, \nu) \leq l_{\infty}(F, \nu)+(d-1) \nu \quad \text { for any } \nu \in \mathbb{Q}, \nu \geq \mathcal{L}(F) .
$$

The proof will be preceded by a lemma.

Lemma 4.7. Let $h \in \mathbb{R}\left[x_{1}, \ldots, x_{n}\right], d=\operatorname{deg} h>0$, and $S=h^{-1}(0)$. If $S \neq \emptyset$, then there exists $C>0$ such that $C|h(x)| \leq \varrho(x, S)\left(1+|x|^{d-1}\right)$ for any $x \in \mathbb{R}^{n}$.

Proof. It is well known that there exist polynomials $h_{1}, \ldots, h_{n} \in \mathbb{R}[x, y]$, where $x=\left(x_{1}, \ldots, x_{n}\right), y=\left(y_{1}, \ldots, y_{n}\right)$, such that $\operatorname{deg} h_{1}, \ldots, \operatorname{deg} h_{n} \leq d-1$ and

$$
h(x)-h(y)=\sum_{j=1}^{n}\left(x_{j}-y_{j}\right) h_{j}(x, y) .
$$

Let $z \in S$. Then there exists $D>0$ such that for any $j=1, \ldots, n$,

$$
\left|h_{j}(x, y)\right| \leq D\left(1+|x|^{d-1}\right) \quad \text { for } x, y \in \mathbb{R}^{n} \text { such that }|y| \leq|z|+2|x| .
$$

Take any $x \in \mathbb{R}^{n}$, and let $y \in S$ be such that $\varrho(x, S)=|x-y|$. Since $\varrho(x, S) \leq|x-z|$, we have $|y| \leq|z|+2|x|$. So, by (25) and (26),

$$
|h(x)| \leq \sum_{j=1}^{n}\left|x_{i}-y_{j}\right|\left|h_{j}(x, y)\right| \leq n|x-y| D\left(1+|x|^{d-1}\right) .
$$

Then, for $C=1 / n D$, we obtain the assertion.

Proof of Corollary 4.6. By Lemma 4.7, there exists $C_{1}>0$ such that

$$
C_{1}|g(x)| \leq \varrho(x, V)\left(1+|x|^{d-1}\right) \quad \text { for } x \in \mathbb{R}^{n} .
$$


Then for $\nu \in \mathbb{Q}$ with $\nu \geq \mathcal{L}(F)$, by Theorem 3.5, there exists $C_{2}>0$ such that

$C_{1}^{\nu} C_{2}|g(x)|^{\nu} \leq C_{2} \varrho(x, V)^{\nu}\left(1+|x|^{d-1}\right)^{\nu} \leq|F(x)|(1+|x|)^{l_{\infty}(F, \nu)}\left(1+|x|^{d-1}\right)^{\nu}$

for any $x \in \mathbb{R}^{n}$. Hence, by Theorem 4.5 , we easily obtain (24).

Acknowledgements. I am deeply grateful to Jacek Chądzyński, Tadeusz Krasiński and Tadeusz Mostowski for their valuable comments and advice.

\section{References}

[1] R. Benedetti and J.-J. Risler, Real Algebraic and Semi-Algebraic Sets, Act. Math., Hermann, Paris, 1990.

[2] H. Bierstone and P. D. Milman, Semi-analytic and subanalytic sets, Publ. I.H.E.S. 67 (1988), 5-42.

[3] J. Bochnak and J.-J. Risler, Sur les exposants de Eojasiewicz, Comment. Math. Helv. 50 (1975), 493-507.

[4] W. D. Brownawell, Local Diophantine Nullstellen inequalities, J. Amer. Math. Soc. 1 (1988), 311-322.

[5] J. Chądzyński, On proper polynomial mappings, Bull. Polish Acad. Sci. Math. 31 (1983), 115-120.

[6] J. Chądzyński and T. Krasiński, Exponent of growth of polynomial mappings of $\mathbb{C}^{2}$ into $\mathbb{C}^{2}$, in: Singularities, S. Eojasiewicz (ed.), Banach Center Publ. 20, PWN, Warszawa, 1988, 147-160.

[7] —, - Sur l'exposant de Eojasiewicz à l'infini pour les applications polynomiales de $\mathbb{C}^{2}$ dans $\mathbb{C}^{2}$ et les composantes des automorphismes polynomiaux de $\mathbb{C}^{2}, \mathrm{C} . \mathrm{R}$. Acad. Sci. Paris 315 (1992), 1399-1402.

[8] -, -, The gradient of a polynomial at infinity, Kodai Math. J. 26 (2003), 317-339.

[9] E. Cygan, A note on separation of algebraic sets and the Eojasiewicz exponent for polynomial mappings, Bull. Sci. Math. 129 (2005), 139-147.

[10] E. Cygan, T. Krasiński and P. Tworzewski, Separation of algebraic sets and the Eojasiewicz exponent of polynomial mappings, Invent. Math. 136 (1999), 75-87.

[11] Z. Denkowska, S. Łojasiewicz and J. Stasica, Certaines propriétés élémentaires des ensembles sous-analytiques, Bull. Acad. Polon. Sci. Math. 27 (1979), 529-535.

[12] A. Fakak, Sur les exposants de Eojasiewicz, Dissertation, Université de Rennes I, 1986, 62 pp. Publ. Inst. Rech. Math. Rennes, 1987-4, Univ. Rennes I, 1988.

[13] A. Gabrielov, Complements of subanalytic sets and existential formulas for analytic functions, Invent. Math. 125 (1996), 1-12.

[14] H. V. Ha, Nombres de Eojasiewicz et singularités à l'infini des polynômes de deux variables complexes, C. R. Acad. Sci. Paris 311 (1990), 429-432.

[15] H. Hironaka, Subanalytic sets, in: Number Theory, Algebraic Geometry and Commutative Algebra, Kinokuniya, Tokyo, 1973, 453-463.

[16] L. Hörmander, On the division of distribution by polynomials, Ark. Mat. 3 (1958), $555-568$.

[17] S. Ji, J. Kollár and B. Shiffman, A global Eojasiewicz inequality for algebraic varieties, Trans. Amer. Math. Soc. 329 (1992), 813-818.

[18] J. Kollár, Sharp effective Nullstellensatz, J. Amer. Math. Soc. 1 (1988), 963-975. 
[19] K. Kurdyka, T. Mostowski and A. Parusiński, Proof of the gradient conjecture of R. Thom, Ann. of Math. 152 (2000), 763-792.

[20] M. Lejeune-Jalabert et B. Teissier, Clôture intégrale des idéaux et équisingularité, Centre de Mathématiques, École Polytechnique, 1974.

[21] S. Łojasiewicz, Sur le problème de la division, Studia Math. 18 (1959), 87-136; and Rozprawy Mat. 22 (1961).

[22] -, Ensembles semi-analytiques, I.H.E.S., Bures-sur-Yvette, 1965.

[23] - Introduction to Complex Analytic Geometry, Birkhäuser, Basel, 1991.

[24] —, Sur la géométrie semi- et sous-analytique, Ann. Inst. Fourier (Grenoble) 43 (1993), 1575-1595.

[25] - On semi-analytic and subanalytic geometry, in: Banach Center Publ. 34, Inst. Math., Polish Acad. Sci., Warszawa, 1995, 89-104.

[26] T. Mostowski, Lipschitz equisingularity, Dissertationes Math. 243 (1985).

[27] -, A criterion for Lipschitz equisingularity, Bull. Polish Acad. Sci. Math. 37 (1989), 109-116.

[28] A. Parusiński, Lipschitz stratification, in: Global Analysis in Modern Mathematics, Proc. of Symposium in Honor of Richard Palais' Sixtieth Birthday, K. Uhlenbeck (ed.), Publish or Perish, Houston, 1993, 73-89.

[29] —, Lipschitz stratification of subanalytic sets, Ann. Sci. École Norm. Sup. 27 (1994), 661-696.

[30] - On the bifurcation set of complex polynomial with isolated singularities at infinity, Compos. Math. 97 (1995), 369-384.

[31] A. Płoski, On the growth of proper polynomial mappings, Ann. Polon. Math. 45 (1985), 297-309.

[32] -, Multiplicity and the Eojasiewicz exponent, in: Banach Center Publ. 20, PWN, 1988, 353-364.

[33] S. Spodzieja, The Eojasiewicz exponent at infinity for overdetermined polynomial mappings, Ann. Polon. Math. 77 (2002), 1-10.

[34] - Eojasiewicz inequality at infinity for the gradient of a polynomial, Bull. Polish Acad. Sci. Math. 50 (2002), 273-281.

Faculty of Mathematics

University of Łódź

Banacha 22

90-238 Łódź, Poland

E-mail: spodziej@math.uni.lodz.pl

Reçu par la Rédaction le 24.11.2004

Révisé le 26.3.2005 